\title{
Review Paper on Fabrication to Improve Process Planning In Rail Bogie
}

\author{
Laxmi Kant Rajak ${ }^{1}$, Novel Kumar Sahu ${ }^{2}$ \\ ${ }^{I} M$-Tech Scholar, Department of Mechanical Engineering, GD Rungta College of Engineering \& Technology \\ Bhilai (C.G.),India \\ ${ }^{I}$ Assistant Professor, Department of Mechanical Engineering, GD Rungta College of Engineering \& \\ Technology Bhilai (C.G.) India
}

\begin{abstract}
This paper presents a study on Process planning is also "the association between product design and fabrication" with the supplementary commission to upkeep design of competitive products. Process planning is of a complex and dynamic nature, often managed by a skilled person with uncommon or no explicit methods to solve the task. In this case Review paper based on the process planning has to be studied and find out the gap which has to be proceeding in further work. The literature review concludes with an overview of the research on strategic planning by intercollegiate athletic departments.
\end{abstract}

Key Words: - Process Planning, Qualitative, Quantitative

\section{Introduction}

Supporting unit was fabricated out of channel sections and equal angles are intended to provide the base of the machine. A bogie is a wheeled wagon or trolley. In terms of mechanics, a bogie is a chassis or framework carrying wheels that are attached to a vehicle, thus serving as a modular subassembly of wheels and axles. Bogies take various forms in various modes of transport. A bogie may remain normally attached on a railway carriage or locomotive or be quickly detachable it may contain a suspensions within it, or be solid and in turn be suspended it may be mounted on a swivel, as on a railway carriage/car or locomotive, or additionally jointed and sprung. The review then narrows the focus to literature on strategic planning in the public sector and the various theories about application of private sector strategic planning to the public organization. The transition from the private sector to the public sector is important because it mirrors the path of adoption for strategic planning to become a management practice in higher education. The literature review then moves to strategic planning in higher education and investigates how higher education institutions are using strategic planning. The review discusses differences in strategic planning and long-range planning and the important role that goals can play in moving from long-range planning to strategic planning.

\section{Literature Review}

Shabana et al. [6] presented a nonlinear finite element formulation for modelling the rail structural flexibility in multibody railroad vehicle systems, it was considered to use two types of interpolations in the kinematic equations developed in the study; the geometry interpolation and the deformation interpolation. The coupling between the rail deformation and geometry, contact coordinates, and non-linear vehicle dynamics was considered. J. Pombo and J. Ambr'osio [16, 19] has developed and implemented a computational tool suitable to study the dynamic behaviour of rail guided vehicles in realistic operation conditions, an efficient multibody methodology was suggested and its computational implementation was discussed. The methodology proposed can be summarized in several points: the description of a three dimensional track model used for a roller coaster application $[18,19]$ and railway vehicle, obtaining realistic track conditions by definition and implementation of the track irregularities; development of a new methodology $[17,20]$ for the accurate prediction of the location of the contact points between the wheel and the rail surfaces; implementation of several creep force models in order to compute all the tangential forces at the contact patch defined in the wheel-rail interaction area; finally validation of the multibody code presented in this work with modeling of a railway vehicle used by Lisbon metro company, and its performance was studied in real operation conditions and in different operation scenarios. The numerical results obtained from the computational tool proposed and the results obtained from ADAMS/RAIL Computer package used to study and simulate the performance of two railway vehicles in real operation conditions, was compared with experimental tests made on the railway vehicles to validate the obtained results.

E. Meli et al. [12] has developed a numerical model which reproduce the complete three-dimensional dynamics of a railway vehicle running on a generic track. The model has been developed with the objective of real-time implementation, in order to use the results to control the actuators of Hardware In the Loop(HIL) test rigs. The numerical model in the test rig has been realised in Matlab-SimulinkTM environment. The module 
was applied to a benchmark vehicle (The Manchester wagon). Comparison between the obtained results and the those obtained using a commercial multibody software package ADAMS/RAIL was shown. In the work presented by Meli et al, it was highlighted that the models used evaluate the deformation of the wheel and the rail in the contact zone by means of two different approaches. With respect to the existing models of railway multibody models, its features were more detailed modeling of wheel-rail contact problems. The step of the track geometry description represents the first step in the solution of the dynamic analysis problem in which the pre-processing operation for the track geometry is made using the input data provided by the manufacturing or the industry to be the input for the track geometry program, then the output data generated in such step provided to the next step which include the multibody dynamic analysis program used to simulate the behavior of the rail guided vehicle. The track model used here in the dynamic simulation must be presented as a parameterized track in order to obtain the required information of the track and all the kinematic and dynamic parameters as a function of the parameter used here which is the distanc covered by the vehicle or the track length, there is two main approaches used in the parameterization of the track center line, the first one uses a combination of analytical segments, straight, transition and plane curve segments to form the track model used in the analysis. The second approach depends on the use of piece wise cubic interpolation schemes to make an interpolation between provided data points representing the track to find the parameterized track center line curve. In both approaches it was necessary to define the cant angle of the track to provide a complete definition of the track. In the methodology presented in the work, it was proposed to use analytical segments to form the presented track, the track model presented in this work consists of tangent or straight line segment, followed by transition curve segment, and finally the plane curve segment with constant radius $\mathrm{R}$. The pre-processed data defined in the track geometry step was provided to a multibody program used for the dynamic analysis of the railroad vehicle, starting with the study and the analysis of a general solid moving along the proposed track. As a next step the analysis of wheelset moving along the track and then the combination between the two solids in the step of the definition of the train vehicle model proposed in the work in the proceeding context.

J. Pombo [18] developed an appropriate methodology for the accurate description of the track centerline geometry, in the frame work of multibody dynamics. A preprocessing step was made to achieve the computational efficiency for the definition of spatial geometry of the centerline based on the data given by the user. Starting with the roller coaster application, four different interpolation schemes was used in the definition of the spatial track centerline. All the information and the data of the right and left rail was stored in a tabulated manner in which interpolation between the entires were made to obtain the required information. The application was extended to be used in the definition of railway track application in which the rail irregularities were implemented and piecewise interpolation schemes were used to parametrize the track irregularities as well as the input data, to obtain the track centerline as a function of the track length.

Shabana et al. [2] use an analytical track description defined by three step procedure: i)Projection, which define the planar curve obtained by projecting the track centerline onto the horizontal plane; ii) Development, which defines an elevation angle; iii) Super-elevation, which defines the track cant angle. In his formulation, a relationship between the arc-length of actual curve and arc-length of the projected curve is stated. Then, the track centerline is defined by providing information about the horizontal curvature as a function of the projected arc-length, the vertical development angle as a function of actual arc-length and the cant angle as a function of the projected arc-length. During the dynamic analysis, the rail space curve are obtained by means of absolute nodal coordinate formulation, leading to an isoparametric beam element that can be conveniently used to describe curved rigid and flexible rails. The method considered each rail as a separate body in order to account for relative motion.

The method used by Shabana [6] in the definition of the track pre-processing step, basically depends on the definition of the geometry file produced, the input data for this program use the industry data such as the curvature, super-elevation and development. The out put data of the pre-processing stage was used in the next stage which is the development of a finite element pre-processor computer program. Description of the rail deformation was discussed which based on the finite element floating frame of reference formulation [3]. The use of this formulation allows for arbitrary rigid body displacement of the track structure, it also allows treating the two rails as one body or two separate bodies. The fundamental component common to all conventional railway vehicles is the wheelset [16]. The movement of the wheelset over the track is characterized by a complex interaction [4] where lateral translation as well as yaw and roll rotations are observed. The formulation of the problem of contact between the wheel and the rail is complex task and has been the subject of several investigations which presented different solutions [5]. Two approaches can be used for solving the problem of wheel-rail contact in railroad dynamics. The first is the commonly called constraint approach, in which nonlinear kinematic contact constraint equations are introduced. In this approach, the contact surfaces are represented in a parametric form using the differential geometry methods. The coordinates of the contact points can be predicted online during the dynamic simulation by introducing surface parameters that describe the contact surface geometries. 
Shabana et al. [5] developed a new elastic force contact formulation for the dynamic simulation of the wheel-rail interaction. In this contact formulation, four surface parameters are introduced in order to be able to describe the geometry of the surfaces of the two bodies that come in contact. The method developed in the mentioned investigation exploits features of multibody computational algorithms that allow adding arbitrary first order differential equations. A differential equation associated with the rail arc length and expressed in terms of the wheel generalized co-ordinates and velocity is used to accurately predict the location of the points of contact between the wheel and the rail. This first order differential equation is integrated simultaneously with the dynamic equations of motion of the wheel-rail system, thereby defining the rail arc length traveled by the wheel. This arc length is used with an optimized search algorithm to determine all possible contact regions.

Pombo [20] presented a new general formulation for the accurate prediction of the location of the contact points on the wheel and rail surfaces. The mentioned model has been proposed and implemented in a general multibody program used in the dynamic analysis of railway vehicles. The coordinates of the contact points are predicted online during the dynamic analysis by introducing the surface parameters that describe the geometry of the contact surfaces. This method was applied to study specific problems inherent to the railway dynamics such as the two points of contact scenario. The methodology to look for the candidates for contact points is fully independent for the wheel tread and for the wheel flange. The used formulation also allowed for investigations related to hunting instability and prediction of wheel climbing, which are very important to study derailment phenomena. The methodology used [16] for the parameterization of the wheel and rail surfaces and for the description of the wheel-rail contact phenomenon was general, since it was able to represent any spatial configuration of the wheels and rails and any wheel and rail profiles, even the ones obtained from direct measurements. Because the wheels are treated separately, the used approach allowed dealing with railway vehicles either with conventional wheelsets, like trains, or with independent wheels, such as in many of the trams in operation.

In the contact model used by E. Meli et al. [12] the contact point position is calculated offline by means of a procedure based on the simplex method. This procedure was used to generate a three dimensional lookup table used in the real-time simulation to find the position of the contact points as a function of wheelset-rail relative displacement, described by three coordinates (the lateral wheelset displacement, the roll and yaw wheelset angle). The procedure was numerically sufficiently efficient and allows multiple contact points to be managed. The method used here in solving the wheelrail contact problem based on the elastic approach, in which the wheel is considered to have six DOF with respect to the rail and the normal contact forces are defined in terms of the indentation between the surfaces and using Hertz contact theory. The main problem encountered in when using the elastic approach, is the determination of the contact points. For sake efficiency, the three dimensional contact problem is usually reduced to a two dimensional problem [12] when searching for the contact points. In the dynamic analysis of railway vehicles, the evaluation of the wheel-rail contact forces is repeated many times. Then, short calculation time algorithm should be used taking the computational cost of the model implemented in the multibody computer program used in the analysis. The method used in the work here for calculating the tangential contact forces and moments is Kalker linear theory of rolling contact [10], this theory based on the assumptions that the existence of small creepages and spin creep, and the area of slip is so small that its influence can be neglected. Under these assumptions, the adhesion zone is assumed to cover the entire area of contact. This method doesn't include the saturation effect of the friction force and, therefore, it is limited to cotact problems with small creepage values. Due to the simplicity and computational implementation easiness, Cartesian coordinates are used [26] in this work to formulate the equations of motion of the multibody systems. No kinematic constraints are added to the formulation, to avoid the complexity produced from the Differential Algebraic Equations (DAE), also the instabilities in the integration process, produced from the substitution of the algebraic equations of the system by their counterpart (ODE), are avoided. Then the equations of motion developed in this work are set of Ordinary Differential Equations (ODE) solved by numerical integration algorithms. Two approaches are often used to formulate the dynamic equations of motion of mechanical systems: the Newtonian and the Lagrangian approaches. In the Newtonian approach, vector mechanics is used to develop the dynamic equations, in this approach the equilibrium position of each body is first studied separately, and it can be used relatively for simple systems and is not suited for the analysis of complex systems such as railroad vehicles. In the Lagrangian approach, scalar quantities such as the virtual work and the kinetic and potential energies are used to develop the equations of motion of the body.

Yaseen \& Wani investigate on Bogie is a chassis carrying wheels attached to the train. Old Bogies are cast models, cast models are huge and bulky. The cast bogie is made in single piece and handling of such components are difficult due to it's over design and manufacturing cost is also high. This project deals with replacing the casted bogie with fabricated bogie by making major changes in design to take the load without failure. The Structural vibrations of the railway bogie are primarily exited by the tracks. These forced vibrations resulting in stress and fatigue of the bogie frame are investigated by simulations. A critical analysis of the railway bogie frame fatigue strength assessment procedure, with special attention to welded joints is carried out. 
By making use of Finite Element program (ANSYS) selected criteria were critically compared for reliability and safety[25]

Avanish et al., plays a key role in transportation of goods and passengers across India. As part of our contribution we have taken up a project related to Indian Railways i.e., to optimize the present bogie design. After completion of this project we will be able to suggest some changes in the design so as to enhance the strength of the bogie and to optimize the material usage in its production. This will reduce the cost of its production. In this regard we have used PRO-E and CATIA and the analysis part is done using HYPERMESH. The main aim of this project is to optimize the design of the bogie of a goods train in order to reduce the material costs without significant reduction in load bearing capacity.[21]

Dooris, Kelley, and Trainer (2002) acknowledge that strategic planning is still relatively new as a management practice. The authors identify the period of time between 1950 and 1970 as the time when strategic planning emerged and note that "the last several decades have been a boom period for strategic planning". As strategic planning has grown in popularity, researchers have devoted more time and attention to defining strategic planning. [10]

Bryson (2004) defines strategic planning as "a disciplined effort to produce fundamental decisions and actions that shape and guide what an organization is, what it does, and why it does it". He says the key to understanding planning is the concept of formalization. He defines strategic planning as "a formalized procedure to produce an articulated result, in the form of an integrated system of decisions". He define strategic planning as "a method used to position an organization, through prioritizing its use of resources according to identified goals, in an effort to guide its direction and development over a period of time". Talk of a "disciplined effort," a "formalized procedure," and "a method," points toward the idea of a process and, as such, the discussion begins with a review of the strategic planning process. Bryson (2004) provides a simple structure for the strategic planning process by defining the ABC's of strategic planning.[9]

Marshall (2004) cautions leaders of higher education institutions that a "cookie-cutter" approach to strategic planning is not effective, noting that, "General prescriptions for 'fixing' higher education rarely work because colleges and universities are complex civic institutions with singular identities"[22]. He support this notion that there is no single best system for planning and suggest that the planning process must be developed specific to the organization to take into account the particular situation and unique characteristics of each organization. He also identify the two major functions of a planning system as developing an integrated, coordinated, and consistent long-term plan of action, and facilitating adaptation of the corporation to environmental change.[22]

Paris (2004) writes, "The process by which the campus strategic plan is developed strongly influences how fully it is implemented". This study, understanding the importance of the strategic planning process, will attempt to identify processes used in strategic planning by intercollegiate athletic departments. In addition to overall process, another common element in the strategic planning literature is the idea of environmental or external scanning. Most authors include the idea of environmental scanning or an environmental assessment as part of the planning process. This environmental scanning component is an important part of strategic planning and helps to distinguish strategic planning from other types of planning.[24]

\section{Conclusion}

Focus is on the underlying process planning efforts needed for its creation. Two research gap are defined as a consequence of the identified problems are:

1. The first gap has an all-machining cost has to reduce, that cover essential activities for process plan synthesis and analysis, including the possibility to predict the outcome of a proposed process plan. These essential activities are all found in the PP flow chart. Much attention is paid to understanding and analysis.

2. The second gap is process sequencing of the request for systematisation and a deeper understanding of process planning, addressed by the academic production research community. The aim here is to develop the PP flow chart on the basis of experiences and findings from the methodology studies.

\section{References}

[1]. Anderberg, S., 2012. Methods for improving performance of process planning for CNC machining - An approach based on surveys and analytical models. Göteborg: Chalmers University of Technology.

[2]. A. A. Shabana, K. E. Zaazaa, and H. Sugiyam. Rail Road Vehicle Dynamics : A Computational Approach. CRC Press, Taylor and Francis group, New York, 2008.

[3]. A. Shabana. Dynamics of Multibody Systems, Second Edition. Cambridge University Press, Cambridge, United Kingdom, 1998 .

[4]. A. Shabana and J. R. Sany. A survey of rail vehicle track simulation and flexible multibody dynamics. Nonlinear Dynamics, 26, 2001 .

[5]. A. A. Shabana, K. E. Zaazaa, J. L. Escalona, and J. R. Sany. Development of elastic force model for wheel/rail contact problems. Journal of Sound and Vibration, 269, 2004.

[6]. A. Shabana, R. Chamorro, and C. Rathod. A multi-body system approach for finite-element modelling of rail flexibility in railroad vehicle applications. Proc. IMechE, Part K: Journal of Multi-body, 222(1), 2008. 
[7]. Bagge, M., Hedlind, M. \& Lindberg, B., 2013. Tolerance chain design and analysis of in-process workpiece. In: A. Archenti \& A. Maffei, eds. Proceedings of the International Conference on Advanced Manufacturing Engineering and Technologies. Stockholm: KTH Royal Institute of Technology, pp. 305-315.

[8]. Bagge, M., Hedlind, M. \& Lindberg, B., 2014. Process chain based workpiece variation simulation for performance utilization analysis. Submitted to Swedish Production Symposium 2014, Göteborg, Sweden, s.n.

[9]. Bryson, J.M. (2004). Strategic planning for public and nonprofit organizations: a guide to strengthening and sustaining organizational achievement (3rd ed.). SanFrancisco: JosseyBass.

[10]. D. Monk-Steel, D. J. Thompson, F. G. de Beer, and M. H. A. Janssens. An investigation into the influence of longitudinal creepage on railway squeal noise due to lateral creepage. Journal of Sound and Vibration, 293, 2006.

[11]. Dooris, M. J., Kelley, J. M., \& Trainer, J. F. (2002). Strategic planning in higher education. New Directions for Higher Education, $116,5-11$.

[12]. E. Meli, M. Malvezzi, S. Papini, L. Pugi, and A. Rindi. A railway vehicle multibody model for real-time application. Vehicle System Dynamics, 46(2), 2008.

[13]. E. Kassa, C. Andersson, and J. C. O. Nielsen. Simulation of dynamic interaction between trainand railway turnout. Vehicle System Dynamics, 44(3), 2006.

[14]. Groche, P. et al., 2012. Integration of manufacturing-induced properties in product design. CIRP Annals - Manufacturing Technology, Volume 61, pp. 163166.

[15]. Gupta, D. P., Gopalakrishnan, B., Chaudhari, S. A. \& Jalali, S., 2011. Development of an integrated model for process planning and parameter selection for machining processes. International Journal of Production Research, 1 November, 49(21), pp. 6301-6319.

[16]. J. Pombo. A multibody methodology for railway dynamics applications. PhD thesis, Instituto Superior T'ecnico, Universidad T’ecnica de Lisboa, 2004.

[17]. J. Pombo and J. Ambr'osio. A computational efficient general wheel-rail contact detection method.

[18]. J. Pombo and J. Ambr'osio. General spatial curve joint for rail guided vehicles: kinematics and dynamics. Multibody System Dynamics, 9, 2003.

[19]. J. Pombo and J. Ambr'osio. A new wheel-rail contact model for railway dynamics. Vehicle System Dynamics, $45(10), 2007$.

[20]. M. Naveen, K. Jagadeshwar, M. Sai Satish Chandra, T. P. S. Avanish, OPTIMIZATION OF A RAILWAY BOGIE, International Journal of Advanced Engineering Technology E-ISSN 0976-3945 IJAET/Vol. IV/ Issue II pp. 71-73, April-June., 2013.

[21]. Marshall, D. R. (2004). Strategic planning: one size doesn't fit all. University Business, 7, 11 - 12.

[22]. Nejad, M. K., Vignat, F. \& Villeneuve, F., 2012. Tolerance analysis in machining using the model of manufactured part (MMP) comparison and evaluation of three different approaches. International Journal of Computer Integrated Manufacturing, 25(2), pp. 136-49.

[23]. Paris, K. A. (2004). Moving the strategic plan off the shelf and into action at the University of Wisconsin-Madison. New Directions for Institutional Research, 123, 121-127.

[24]. Syed Yaseen \& Trupti Wani, Design and Analysis of a Railway Bogie Truck, International Journal of Ignited Minds (IJIMIINDS) Volume: 01 Issue: 06 pp. 13-19, June-2014

[25]. W. Zhai, K. Wang, and C. Cai. Fundamentals of vehicle-track coupled dynamics. Vehicle System Dynamics, 47(11), 2009.

[26]. Werke, M., Bagge, M., Nicolescu, M. \& Lindberg, B., 2014. Process modelling using upstream analysis of manufacturing sequences. The International Journal of Advanced Manufacturing Technology, Volume Under review April 2014.

[27]. Wu, C. -W., Pearn, W. L. \& Kotz, S., 2009. An over view of theory and practice on process capability indices for quality assurance. International Journal of Production Economics, Volume 117, pp. 338-359.

[28]. Xu, H.-M., Yuan, M.-H. \& Li, D.-B., 2009. A novel process planning schema based on process knowledge customization. International Journal of Advanced Manufacturing Technology, Volume 44, pp. 161-172.

[29]. Xu, X., Wang, L. \& Newman, S. T., 2011. Computer-aided process planning - A critical review of recent developments and future trends. International Journal of Computer Integrated Manufacturing, January, 24(1), pp. 1-31. 